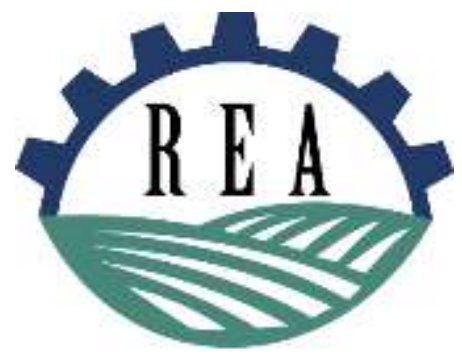

Revista de Economia e Agronegócio - REA ISSN impresso: 1679-1614 ISSN online: $2526-5539$ Vol. 16 | N. 2 | 2018

Camila Stefaní de Sousa Silva' Cassiano Bragagnolo ${ }^{1^{*}}$

1 Universidade Federal de São Carlos, Departamento de Economia, Sorocaba, SP, Brasil

* cassiano@ufscar.br

\section{EFICIÊNCIA TÉCNICA E AMBIENTAL DA PECUÁRIA LEITEIRA NA REGIÃO DO TRIÂNGULO MINEIRO E ALTO PARANAÍBA}

RESUMO

A fermentação entérica do gado de corte e leite é o principal responsável pela emissão de metano no Brasil. Em Minas Gerais, a agropecuária é a atividade com maior estimativa de emissão de gases de efeito estufa. Apesar desses impactos ambientais negativos, essa atividade possui grande importância econômica para diversas regiões, entre as quais o Triângulo Mineiro e Alto Paranaíba, em Minas Gerais. Assim, o presente trabalho estimou uma função de produção, por meio de análise econométrica de fronteira estocástica, visando a avaliar a eficiência técnica e ambiental na produção de leite na região do Triângulo Mineiro e Alto Paranaíba. Para estimar as fronteiras de eficiência técnica e ambiental, foi utilizada a metodologia exposta por Reinhard, Lovell e Thijssen (1999). A base de dados adotada foi disponibilizada pelo projeto Educampo/SEBRAE, com informações de 178 produtores de leite da região analisada para o ano de 2016. Os resultados apontaram que a média da eficiência técnica obtida pelas fazendas observadas foi de $89 \%$, enquanto a da eficiência ambiental foi de $73 \%$. Além disso, através da correlação de Spearman, verificou-se que, conforme esperado, há uma relação positiva entre as eficiências técnica e ambiental.

Palavras-chave: Produção de Leite; Economia Regional; Eficiência Técnica; Eficiência Ambiental.

\section{ABSTRACT}

The cattle enteric fermentation is an important part of the methane emissions in Brazil. In Minas Gerais state, agriculture is the activity with the highest estimate of greenhouse gases emissions. Despite these negative environmental effects, milk production is an important economic activity for several regions, among which the Triângulo Mineiro and Alto Paranaíba in Minas Gerais. This paper estimates a production function, through stochastic frontier econometric analysis, aiming to evaluate the technical and environmental efficiency in milk production in the Triângulo Mineiro and Alto Paranaíba region. To estimate the boundaries of technical and environmental efficiency, we used the methodology presented by Reinhard, Lovell and Thijssen (1999). The database are from Educampo/SEBRAE project with information from 178 dairy farms for 2016. The results showed that the average of the technical efficiency was $89 \%$, while the environmental efficiency was $73 \%$. In addition, analyzing Spearman's correlation, there is a positive relationship between technical and environmental efficiencies.

Keywords: Milk Production; Regional Economy; Technical Efficiency; Environmental Efficiency.

JEL Code: Q12; Q54; R11. 



\section{INTRODUÇÃO}

O agronegócio é uma das principais atividades econômicas do Brasil. Em 2015, o Produto Interno Bruto (PIB) dessa atividade foi de R\$1,3 trilhão, sendo que desse montante o PIB da pecuária foi de R 409 bilhões, contribuindo de forma expressiva para o valor adicionado da economia brasileira (CEPEA, 2016). Estima-se que o Brasil tenha mais de um milhão e trezentas mil propriedades que produzem leite, ocupando, diretamente, cerca de 3,6 milhões de pessoas (CARVALHO et al., 2003; IBGE, 2006). A despeito da importância econômica e social para o Brasil, as atividades agropecuárias têm recebido críticas em relação aos efeitos ambientais adversos gerados por elas (CAMPOS, 2011).

Entre esses efeitos ambientais, pode-se citar a emissão de gases poluentes de efeito estufa, como o metano $\left(\mathrm{CH}_{4}\right)$, o dióxido de carbono $\left(\mathrm{CO}_{2}\right)$ e o óxido nitroso $\left(\mathrm{S}_{2} \mathrm{O}\right)$. Além disso, esses gases provocam externalidades negativas na produção, gerando problemas ambientais, como eutrofização das águas superficiais, lixiviação de nitratos nos lençóis freáticos e formação de chuvas ácidas (REINHARD, LOVELL; THIJSSEN, 1999). Pode-se citar também, como agravantes ambientais, o desmatamento, a erosão, as queimadas e a desertificação, provenientes do manejo inadequado das pastagens utilizadas na atividade.

Dados do Inventário Brasileiro de Emissões indicam que a agropecuária brasileira, em 2014, foi responsável por emitir 33\% do total de gases de efeito estufa no país. Na agropecuária, a fermentação entérica, fonte elementar de produção de gás metano por parte do gado bovino, e a aplicação de adubos e fertilizantes sintéticos, capaz de gerar óxido nitroso, foram os principais meios de emissão desses gases (MCTI, 2016).

Primavesi et al. (2004) destacam que países em desenvolvimento localizados em regiões tropicais apresentam altos índices de emissão de gases de efeito estufa, visto que as condições climáticas dessas localidades aumentam o potencial de emanação de gases como o metano. No Brasil, em 2014, as emissões de metano pelo setor agropecuário ocorreram em maior proporção pela fermentação entérica do gado de corte e leite, representando $87 \%$ do total de emissões desse gás. Os 13\% restantes foram provenientes do manejo de dejetos de criações de bovinos, de suínos, de aves e outros, além da produção de cana-de-açúcar e do cultivo de arroz (MCTI, 2016).

Em Minas Gerais, a agropecuária é a atividade com maior estimativa de emissão de gases de efeito estufa. Em 2014, esse setor foi responsável por $41 \%$ do total das emissões do estado. Entre 2005 e 2014, as emissões do setor aumentaram em 15\%. Justifica-se esse aumento pelo crescimento do rebanho bovino no estado. Além disso, a pecuária é uma das principais atividades responsáveis pela emissão de metano, que, através da 
fermentação entérica, em 2014, gerou 26,5 $\mathrm{MtCO}_{2} e q / a n o^{1}$. O manejo de dejetos, por sua vez, emitiu 2,53 $\mathrm{MtCO}_{2}$ eq/ano ${ }^{2}$ (FEAM, 2016).

A despeito dos problemas ambientais gerados, o leite é um dos principais produtos da agropecuária brasileira. A atividade em torno da produção de leite desempenha uma função importante no desenvolvimento econômico e social do país, principalmente na geração de emprego e renda (Carvalho et al., 2003).

A produção de leite bovino no Brasil, em 2015, foi de 35 bilhões de litros. Minas Gerais foi o maior produtor do país com 9 bilhões de litros, o que corresponde a $26 \%$ do total nacional. No estado, a mesorregião com maior produção é o Triângulo Mineiro e Alto Paranaíba, com uma participação de $25 \%$ de todo o volume produzido em Minas Gerais, equivalente a 2,4 bilhões de litros de leite (IBGE, 2016).

De acordo com dados do IBGE (2016), no ano de 2015, o efetivo de bovinos brasileiro foi de 215 milhões de cabeças, sendo o maior rebanho comercial do mundo. Desse total, 21 milhões de cabeças correspondem a vacas de ordenha. Em Minas Gerais, o efetivo de bovinos, em 2015, foi de 23 milhões de cabeças, e desse montante, 5,4 milhões são vacas de ordenha. Ressalta-se que a região do Triângulo Mineiro e Alto Paranaíba detém o maior efetivo de bovinos do estado, com cerca de 6 milhões de cabeças e 1,2 milhões de vacas de ordenha.

Com base na importância da produção de leite e nos problemas ambientais advindos dela, este trabalho tem por objetivo analisar a eficiência técnica e ambiental da bovinocultura leiteira na mesorregião do Triângulo Mineiro e Alto Paranaíba, em Minas Gerais. Com isso, pretende-se averiguar a possibilidade de uma produção eficiente do ponto de vista econômico, alinhada a uma atividade com menores danos ao meio ambiente. Por fim, busca-se avaliar a correlação entre o desempenho técnico e o ambiental. Para tanto, uma base de dados com informações de 178 produtores de leite da região analisada, fornecida pelo projeto Educampo/SEBRAE para o ano de 2016, será utilizada.

No Brasil, grande parte das políticas públicas voltadas para o setor agrícola estão relacionadas ao aumento de produtividade das atividades agropecuárias. Observa-se que a maior parte dos estudos disponíveis na literatura buscam avaliar a eficiência técnica gerando, portanto, uma escassez de trabalhos sobre eficiência ambiental. Embora haja uma insuficiência de pesquisas a respeito do tema, as preocupações com o meio ambiente e a emissão de gases poluentes são tão relevantes quanto a análise do desempenho técnico da pecuária leiteira, uma vez que as emissões de

\footnotetext{
${ }^{1} \mathrm{CO}_{2}$ eq é uma unidade de medida utilizada para expressar a quantidade de gases de efeito estufa emitidos de forma equivalente à quantidade de dióxido de carbono (MCTI, 2014). Nesse caso, $\mathrm{MtCO}_{2}$ eq/ano significa milhões de toneladas de dióxido de carbono equivalente.

2 Apesar de as emissões decorrentes do manejo de dejetos serem menores do que as ocasionadas pela fermentação entérica, esta representa uma fonte importante de emissão quando somada aos lançamentos desses dejetos no solo (FEAM, 2016).
} 
gases de efeito estufa impactam a dinâmica produtiva do setor. Assim, considera-se a importância de se estabelecer uma relação harmônica no âmbito do processo de uma atividade produtiva tão relevante economicamente como a produção de leite, principalmente em Minas Gerais, especificamente no Triângulo Mineiro e Alto Paranaíba, com o cumprimento de medidas que visam a garantir qualidade ambiental.

Diferentemente dos demais estudos disponíveis na literatura, este tem foco na emissão de metano e não de nitrogênio, visto que a emissão desse gás pelo rebanho bovino é expressiva e considerada um problema ambiental grave.

Este trabalho está organizado em cinco partes, a começar com esta breve introdução ao tema, em que se apresenta a justificativa e os objetivos deste artigo. A segunda parte trata-se de um breve referencial teórico, com conceitos e estudos referentes à eficiência técnica e ambiental. A seção seguinte exibe o método empregado e a construção do modelo utilizado. A quarta parte apresenta e discute os resultados obtidos. Por fim, a quinta parte é referente às considerações finais deste artigo, em que são feitas reflexões gerais sobre a pesquisa, além de abordar suas contribuições e limitações.

\section{REVISÃO DE LITERATURA}

Em seu trabalho pioneiro, Reinhard, Lovell e Thijssen (1999) propuseram o cálculo das eficiências técnica e ambiental para um painel de fazendas de gado leiteiro na Holanda, buscando averiguar sua variação ao longo do tempo e se havia compatibilidade entre os resultados obtidos. No artigo, Reinhard, Lovell e Thijssen (1999) tratam o excedente de nitrogênio, decorrente da aplicação de quantidades excessivas de esterco e fertilizante químico, como um insumo ambientalmente prejudicial (detrimental input). Uma fronteira estocástica de produção translog foi especificada para estimar a eficiência técnica orientada para a produção. A eficiência ambiental é definida como a relação entre o uso mínimo viável e o uso observado de um insumo ambientalmente prejudicial, condicionada aos níveis constatados do produto desejado e dos insumos convencionais. Foi utilizado como insumo ambientalmente prejudicial o excedente de nitrogênio de cada fazenda, que decorre da aplicação de quantidades excessivas de esterco e fertilizantes químicos. A eficiência técnica média foi elevada, de 0,894. A eficiência ambiental média, porém, foi de apenas 0,441. Além disso, os autores destacam que as fazendas leiteiras intensivas apresentaram resultados técnica e ambientalmente mais eficientes do que as fazendas extensivas (REINHARD, LOVELL; THIJISSEN, 1999).

Gourley, Aarons e Powell (2012) verificaram que o aumento da produção de produtos lácteos no mundo foi acompanhado pelo crescimento no uso de insumos compostos por nitrogênio para obter ganhos de produtividade. $\mathrm{O}$ acréscimo no uso desses insumos gerou taxas maiores de excesso de nitrogênio e, por conseguinte, degradação ambiental. Assim, os autores avaliaram a eficiência do uso de nitrogênio em compostos alimentares e nas 
concentrações de ureia no leite das vacas em lactação, bem como nas práticas de manejo do estrume em 29 fazendas em Victoria, na Austrália, e em Wisconsin, nos EUA. Desse modo, o estudo objetivou identificar o melhor desempenho no uso do nitrogênio, pois melhorias nas práticas alimentares do rebanho e no manejo dos adubos beneficiariam a indústria do setor, aperfeiçoando tanto seu desempenho técnico quanto os efeitos ambientais. Os resultados obtidos pelos autores demonstraram que, nas fazendas das cidades analisadas, independente do sistema de produção adotado, somente uma pequena parcela das terras disponíveis foram utilizadas para depositar o nitrogênio excretado. $\mathrm{Na}$ maioria das propriedades, quantidades significativas do estrume coletado foram colocadas em áreas não produtivas, aumentando, assim, o risco de perdas de nitrogênio por escoamento superficial (GOURLEY, AARONS; POWELL, 2012).

Segundo Berre et al. (2014), há uma tendência a avaliar as políticas de produção pecuária considerando os efeitos da atividade no meio ambiente. Dessa forma, o autor centrou seu estudo na análise do trade-off entre a produção de leite e seu efeito ambiental, considerando as emissões de gases do efeito estufa, mais precisamente de nitrogênio. Sendo assim, por meio do método de análise envoltória dos dados (DEA), foi possível demonstrar qual a melhor maneira de reduzir a ineficiência ambiental. No melhor sistema, foi possível reduzir as emissões de dióxido de carbono em 238 gramas por litro de leite, o que corresponde a um valor 13,93\% menor do que o valor inicial. Além disso, conseguiu-se aumentar a produção de leite em 7,72 litros de leite para cada quilo de excedente de nitrogênio.

Nijuki e Bravo-Ureta (2015) analisaram o impacto econômico associado a um regime regulatório ambiental hipotético que possui o objetivo de reduzir as emissões dos gases de efeito estufa em alguns municípios produtores de leite dos Estados Unidos. Para tal, foi estabelecido um índice de poluição amplo que combina gado, combustível e fontes de fertilizantes responsáveis pela poluição. $\mathrm{O}$ método se diferencia do proposto por Reinhard, Lovell e Thijssen (1999), em que foi considerada apenas uma medida parcial com base no excesso de emissão de nitrogênio. Identificouse que os estabelecimentos que cumprem as exigências da regulamentação apresentam ganhos de 5\% na eficiência técnica média. Os custos decorrentes das novas exigências variam, sendo que as produções que operam em maior escala apresentam maior redução de gastos.

Dayananda (2016) utilizou um modelo de fronteira de eficiência estocástica para estimar a eficiência técnica e ambiental de 143 fazendas leiteiras localizadas em Ontário no Canadá. As fazendas analisadas possuem bons índices de eficiência técnica, com média de $82 \%$. Em contrapartida, a média de eficiência ambiental verificada é baixa, sendo de $30 \%$. Além disso, foi verificado que quanto maior o rebanho, maiores são os índices de eficiência ambiental. Por fim, foi averiguado que há uma correlação positiva entre as duas eficiências, ou seja, fazendas com elevado nível de eficiência técnica tendem a ter um elevado nível de eficiência ambiental. 
Coelli, Lauwers e Huylenbroeck (2006) propuseram uma abordagem diferente daquelas dos demais autores citados para o cálculo da eficiência ambiental, incorporando o conceito de equilíbrio de materiais no modelo. Dessa maneira, a estimativa de eficiência ambiental proposta pelos autores pôde ser decomposta em eficiências técnica e alocativa, de forma equivalente à decomposição convencional de eficiência técnica.

Para o Brasil, Campos (2011) desenvolveu pesquisa similar àquela que se pretende desenvolver neste trabalho. $\mathrm{O}$ autor estimou as eficiências técnica e ambiental, de curto e de longo prazo, para as unidades produtoras de leite de Minas Gerais no ano de 2005. A metodologia foi baseada nos modelos teóricos de Barua et al. (2004) e Coelli, Lauwers e Huylendroeck (2006). Para estimar a eficiência ambiental, o autor analisou as emissões de nitrogênio provenientes da produção leiteira. Os resultados obtidos demonstram que uma redução da emissão de nitrogênio implica um aumento dos custos de produção, visto que para obter tal objetivo os insumos utilizados deveriam ser substituídos por outros de maior valor.

\section{METODOLOGIA E BASE DE DADOS}

\section{Modelo de fronteira estocástica e a eficiência ambiental}

Optou-se pelo método de fronteira estocástica para o desenvolvimento deste trabalho, devido ao fato de esse procedimento ter a vantagem de distinguir os efeitos do erro estatístico daqueles da ineficiência produtiva, ou seja, nesse tipo de modelo, os desvios da fronteira podem não estar inteiramente sob o controle das unidades produtivas estudadas. Dentre as vantagens de se utilizar essa metodologia, pode-se destacar a possibilidade de especificar hipóteses e a construção de intervalos de confiança. Destacase ainda que, de acordo com Coelli (1995), o método de fronteira estocástica é recomendado para estudo de unidades produtivas agrícolas. Isso se deve ao fato de que erros de medição, omissão de variáveis e a influência climática podem interferir no resultado agrícola. Devido a essas interferências, não é recomendado que todos os desvios da fronteira sejam relacionados à ineficiência.

A análise de fronteira estocástica é uma abordagem analítica que utiliza técnicas econométricas. Nesse modelo, incorporam-se a ineficiência técnica, o controle dos produtores e os choques aleatórios como meios de interferência na quantidade produzida. Assim, pode-se representar um modelo de fronteira de produção de acordo com a eq. (1) (KUMBHAKAR; LOVELL, 2000).

$$
Y_{i}=f\left(X_{i} ; \beta\right) . T E_{i}
$$

em que $Y_{i}$ é o produto da unidade de produção i, com i $=1,2, \ldots, \mathrm{n} ; X_{i}$ é um vetor de $n$ insumos; $f\left(X_{i} ; \beta\right)$ é a fronteira de produção e $\beta$ é o vetor de parâmetros a ser estimado. A expressão $T E_{i}$ denota a eficiência técnica produto-orientada de cada produtor e é representada pela eq. (2).

Revista de Economia e Agronegócio - REA | V. 16 | N. 2 | 2018 | pág. 247 


$$
T E_{I}=\frac{Y_{i}}{f\left(X_{i} \beta\right)}
$$

A eq. (2) é a razão entre o produto observado e o produto máximo produzido. Assim, $Y_{i}$ alcançará seu valor máximo de $f\left(X_{i} ; \beta\right)$ se $T E_{i}=1$. A diferença entre $Y_{i}$, que é uma medida observada, e a produção máxima possível $f\left(X_{i} ; \beta\right)$ é denominada ineficiência técnica.

Para incorporar a parte estocástica à expressão $f\left(X_{i} ; \beta\right)$, utiliza-se a eq. (3).

$$
Y_{i}=f\left(X_{i} ; \beta\right) \cdot \exp \left\{v_{i}\right\} \cdot T E_{i}
$$

na qual $f\left(X_{i} ; \beta\right) \cdot \exp \left\{v_{i}\right\}$ representa a fronteira de produção estocástica. A variável $\exp \left\{v_{i}\right\}$ capta os efeitos dos choques aleatórios, que podem ser atribuídos à variação do trabalho, ao desempenho do maquinário, aos fenômenos climáticos, entre outros. Assim, tem-se a relação descrita pela eq. (4).

$$
T E_{i}=\frac{Y_{i}}{f\left(X_{i} ; \beta\right) \cdot \exp \left\{v_{i}\right\}}
$$

A eq. (4) é a razão entre o produto observado e a produção máxima, dado os choques aleatórios $\exp \left\{v_{i}\right\}$. A variável $Y_{i}$ atinge valor máximo quando $T E_{i}=1$.

Conforme demonstrado por Reinhard, Lovell e Thijssen (1999), a fronteira de produção estocástica com insumos ambientalmente nocivos pode ser representada pela eq. (5).

$$
Y_{i}=F\left(X_{i}, Z_{i}, \beta\right) \cdot \exp \left\{v_{i}-u_{i}\right\}
$$

em que $Y_{i}$ é o nível de produção, $X_{i}$ é o vetor de insumos que não trazem prejuízo ao meio ambiente - denominados pelos autores como conventional inputs - e a variável $Z_{i}$ representa o insumo que prejudica o meio ambiente - que os autores chamam de detrimental inputs.

A eficiência ambiental é alcançada quando se utiliza a menor proporção de detrimental inputs para se obter a maior quantidade de produto possível. Embora os efeitos nocivos das emissões de gases sejam difíceis de quantificar, os insumos que geram esses efeitos podem ser quantificados e analisados de acordo com o desempenho econômico e ambiental (REINHARD, LOVELL e THINJSSEN, 1999). Assim, neste estudo, a eficiência ambiental será mensurada como em Reinhard, Lovell e Thinjssen (1999), em que o excedente de nitrogênio, apesar de ser tratado como um insumo prejudicial ao meio ambiente, é inserido na função de produção translog e analisado como um insumo comum. Esse procedimento pode ser justificado pelo fato de que a produção de produtos indesejáveis cresce com o aumento de bens. Assim, frequentemente argumenta-se que a relação monotônica entre os bens e os produtos indesejáveis é similar à relação entre insumos e bens. Dessa maneira, vários autores, entre os quais pode-se citar Reinhard, Lovell e Thijssen $(1999,2000)$ e Lansink, Pietola e Bäckman 
(2007), tratam da poluição gerada no processo por meio da modelagem do uso dos insumos.

Embora seja prática habitual na literatura, o tratamento de produtos indesejáveis como insumos tem sido criticado por autores como Hailu e Veeman (2001), Lee, Park e Kim (2002) e Färe et al. (2005). Assim, faz-se necessário evidenciar que, embora a metodologia adotada nesta pesquisa se baseie no modelo teórico de Reinhard, Lovell e Thijssen (1999) e seja recorrente na literatura, o insumo prejudicial adotado corresponde à emissão de metano sendo tratado como um input, o que pode ser encarado como uma limitação.

Diferentemente de alguns modelos, que inserem a variável que prejudica o meio ambiente como um produto indesejável, o utilizado neste estudo a estabelece como um insumo. $\mathrm{O}$ vetor de parâmetros que será estimado é representado por $\beta$. O termo de erro possui dois componentes, $v_{i}$ e $u_{i}$. O termo $v_{i}$ é simétrico e sua distribuição ocorre independente de $u_{i}$. Sendo assim, caso $u_{i} \geq 0$, o termo de erro $\varepsilon_{i}=v_{i}-u_{i}$ será assimétrico.

Desse modo, a medida de eficiência técnica estocástica (TE) produtoorientada é expressa pela eq. (6).

$$
T E=\frac{Y_{i}}{\left[F\left(X_{i}, Z_{i ;} \beta\right) \cdot \exp \left\{v_{i}\right\}\right]}=\left\{\exp -u_{i}\right\}
$$

A eq. (6) considera que $u_{i} \geq 0,0 \leq \exp \left\{-u_{i}\right\} \leq 1$. A ineficiência técnica é representada pela diferença entre os termos de erro $\left(v_{i}-u_{i}\right)$.

A fronteira de produção estocástica em formato translog pode ser representada pela eq. (7).

$$
\begin{aligned}
& \ln Y_{i}=\beta_{0}+\sum_{j} \beta_{j} \ln X_{i j}+\beta_{z} \ln Z_{i t}+ \\
& \frac{1}{2} \sum_{j} \sum_{k} \beta_{j k} \ln X_{i j} \ln X_{i k}+\sum_{j} \beta_{j z} \ln X_{i j} \ln Z_{i}+\frac{1}{2} \beta_{z z}\left(\ln Z_{i}\right)^{2}+v_{i}-u_{i}
\end{aligned}
$$

A eficiência técnica é obtida quando $u_{i}=0$, já a eficiência ambiental é atingida quando se substitui $Z_{i t}$ por $Z_{i t}^{F}$, e definindo $u_{i}=0$ na eq. (7) (REINHARD, LOVELL e THIJSSEN, 1999).

$$
\begin{aligned}
& \ln Y_{i}=\beta_{0}+\sum_{j} \beta_{j} \ln X_{i j}+\beta_{z} \ln Z_{i}^{F}+\frac{1}{2} \sum_{j} \sum_{k} \beta_{j k} \ln X_{i j} \ln X_{i k}+ \\
& \sum_{j} \beta_{j z} \ln X_{i j} Z_{i}^{F}+\frac{1}{2} \beta_{z z}\left(\ln Z_{i}^{F}\right)^{2}+v_{i t}
\end{aligned}
$$

A mensuração da forma logarítmica da medida de eficiência ambiental, representada por $\ln E E_{i}=\ln Z_{i}^{F}-\ln Z_{i}$, pode ser obtida ao se igualar as eq. (7) e (8). Segundo Reinhard, Lovell e Thijssen (1999), ela poderá ser expressa, portanto, de acordo com a eq. (9).

$$
\begin{gathered}
\frac{1}{2} \beta_{z z}\left[\left(\ln Z_{i}^{F}\right)^{2}-\left(\ln Z_{i}\right)^{2}\right]+\sum_{j} \beta_{j z} \ln X_{i j}\left[\ln Z_{i}^{F}-\ln Z_{i}\right] \\
+\beta_{z}\left[\ln Z_{i}^{F}-\ln Z_{i}\right]+u_{i}=0
\end{gathered}
$$


Reescrevendo a eq. (9) e isolando $\ln E E_{i}=\ln Z_{i}^{F}-\ln Z_{i}$, a eficiência ambiental pode ser estimada por meio da raiz quadrada positiva da eq. (10) - resultado este conhecido como $+\sqrt{ }$ fórmula (REINHARD, LOVELL; THIJSSEN, 1999).

$$
\begin{aligned}
& \ln E E_{i}=\left[-\left(\beta_{z}+\sum_{j} \beta_{j z} \ln X_{i j}+\beta_{z z} \ln Z_{i}\right) \pm\left\{\left(\beta_{\mathrm{z}}+\sum_{\mathrm{j}} \beta_{\mathrm{jz}} \ln \mathrm{X}_{\mathrm{ij}}+\right.\right.\right. \\
& \left.\left.\left.\beta_{\mathrm{zz}} \ln \mathrm{Z}_{\mathrm{i}}\right)^{2}-2 \beta_{\mathrm{zz}} u_{\mathrm{i}}\right\}^{0,5}\right] / \beta_{\mathrm{zz}}
\end{aligned}
$$

Assim, a fronteira de eficiência técnica e ambiental pode ser estimada tanto por modelos determinísticos quanto estocásticos, porém a segunda opção pode vir a produzir estimativas mais precisas.

Foi utilizado nas estimações o método de máxima verossimilhança, proposto por Aigner, Lovell e Schmidt (1977). Esse modelo permite separar o termo de erro em dois componentes com mais facilidade, se comparado com o método de mínimos quadrados ordinários.

Dessa forma, para o cálculo da eficiência técnica, este trabalho utiliza uma função translog, expressa pela eq. (11).

$$
\begin{aligned}
& \text { lny }_{i}=\beta_{0}+\beta_{1} \text { lnarea }_{i}+\beta_{2} \text { lntrab }_{i}+\beta_{3} \text { lnalimen }_{i}+\beta_{4} \text { lncap }_{i} \\
& +\beta_{5} \operatorname{lnz}_{i}+\frac{1}{2}\left[\beta_{6}\left(\text { lnarea }_{i}\right)^{2}+\beta_{7}\left(\text { lnarea }_{i} x \operatorname{lntrab} b_{i}\right)\right. \\
& +\beta_{8}\left(\text { area }_{i} x \text { lnalimen }_{i}\right)+\beta_{9}\left(\text { lnarea }_{i} x \operatorname{lncap}_{i}\right) \\
& +\beta_{10}\left(\text { lnarea }_{i} x \ln z_{i}\right)+\left(\beta_{11}\left(\text { lntrab }_{i}\right)^{2}\right. \\
& +\beta_{12}\left(\text { lntrab }_{i} x \operatorname{lnalimen}_{i}\right)+\beta_{13}\left(\operatorname{lntrab}_{i} x \operatorname{lncap}_{i}\right) \\
& +\beta_{14}\left(\operatorname{lntrab}_{i} x \ln z_{i}\right)+\beta_{15}\left(\text { lnalimen }_{i}\right)^{2} \\
& +\beta_{16}\left(\text { lnalimen }_{i} x \operatorname{lncap}_{i}\right)+\beta_{17}\left(\text { lnalimen }_{i} x \ln z_{i}\right) \\
& +\beta_{18}\left(\operatorname{lncap}_{i}\right)^{2}+\beta_{19}\left(\operatorname{lncap}_{i} x \ln z_{i}\right)+\beta_{20}\left(\ln z_{i}\right)^{2}+v_{i} \\
& -u_{i}
\end{aligned}
$$

em que $y_{i}$ refere-se à quantidade anual de leite produzido, medida em litros; are $_{i}$ se refere ao tamanho da propriedade, medido em hectares; $\operatorname{trab}_{i}$ representa o custo com mão de obra despendido na produção; alimen $_{i}$ é a soma das despesas com concentrado e volumosos; $\operatorname{cap}_{i}$ representa o capital utilizado na produção - para obtê-lo, foi realizado o somatório dos valores dos estoques de benfeitorias, de forrageiras, de máquinas e de animais -, e $z_{i}$ representa o insumo ambiental indesejado, que, neste trabalho, corresponde à emissão de metano.

Após estimar os parâmetros do modelo, são calculados os níveis de eficiência técnica de cada uma das fazendas analisadas. Os valores de eficiência variam entre 0 e 1 e quanto mais próximo a 1 , mais eficiente é a produção. Além disso, calculam-se também os níveis de eficiência ambiental das fazendas. Para esse cálculo, utilizou-se os termos de erro e os parâmetros relacionados ao insumo correspondente à emissão de metano, de acordo com o procedimento descrito na eq. (10). 


\section{Base de dados}

Os dados utilizados nesta pesquisa se referem às unidades produtoras de leite do Triângulo Mineiro e Alto Paranaíba participantes do projeto Educampo, que teve início em 1977 no estado de Minas Gerais e é incentivado pelo Serviço Brasileiro de Apoio às Micro e Pequenas Empresas (SEBRAE). Seu objetivo é prestar assistência gerencial e tecnológica para produtores rurais do estado, com o intuito de auxiliar na administração das propriedades.

As informações econômicas e técnicas utilizadas se referem a 178 propriedades do Triângulo Mineiro e Alto Paranaíba pertencentes ao projeto no ano de 2016. A base de dados do Educampo foi escolhida por oferecer informações detalhadas sobre as propriedades de leite de Minas Gerais e por conter dados de períodos recentes. A amostra contém informações econômicas das fazendas, sobre custos dos insumos, recursos disponíveis para a produção e tecnologias utilizadas. Dessa forma, foi possível analisar a eficiência técnica e a ambiental.

O modelo estimado tem como variável dependente a quantidade, em litros, de leite produzido, e como insumos convencionais utilizou-se a área da propriedade, medida em hectares, as despesas com mão de obra e alimentação e o estoque de capital. Para calcular o estoque de capital, utilizou-se como proxy o valor dos bens utilizados na produção de leite nas unidades produtivas, que foram declarados pelos produtores na pesquisa. Assim, somou-se os valores das benfeitorias, das forrageiras, das máquinas e implementos e dos animais.

Além disso, utilizou-se a variável relativa à emissão de metano como insumo ambiental não desejável (detrimental input). Para estimar a emissão de metano nas unidades produtivas, utilizou-se o método "Tier 2" do Intergovernmental Panel on Climate Change (IPCC). A metodologia em questão pode ser encontrada, detalhadamente, em IPCC (2006).

\section{RESULTADOS E DISCUSSÃO}

A Tabela 1 apresenta as estatísticas descritivas das variáveis utilizadas na estimação do modelo. Ao observar a variável relativa à produção de leite $\left(y_{i}\right)$, verifica-se que a quantidade média produzida pelas fazendas analisadas foi de 436 mil litros por ano. O valor médio despendido com a mão de obra foi de R\$ 69 mil por ano e o valor médio do estoque de capital foi de R\$ 762 mil. A despesa média com alimentação do rebanho, em 2016, foi de R $\$ 318$ mil. Ao analisar a Tabela 1, percebe-se que o custo médio com esse item é superior às despesas com mão de obra. Esse resultado está em conformidade com o que foi exposto pelo International Farm Comparison Network (IFCN, 2014), no sentido de que o valor despendido com a alimentação do animal é um dos itens que mais eleva os custos de produção. Assim, quanto maior a qualidade do alimento oferecido ao rebanho mais custosa será a produção. Por fim, a emissão de metano média foi de 0,01 $\mathrm{GgCO}_{2} e q$. 
Tabela 1. Estatísticas descritivas das variáveis utilizadas

\begin{tabular}{ccccccc}
\hline Variável & Unidade & Observação & Média & $\begin{array}{c}\text { Desvio- } \\
\text { padrão }\end{array}$ & Mínimo & Máximo \\
\hline$y_{i}$ & Litros & 178 & 436.742 & 426,10 & 550.72 & 4.144 .363 \\
area $_{i}$ & Hectares & 178 & 69,86 & 62,00 & 6,43 & 526,92 \\
rrab $_{i}$ & $\mathrm{R} \$$ & 178 & 70.721 & 49.846 & $8.219,61$ & $365.421,84$ \\
cap $_{i}$ & $\mathrm{R} \$$ & 178 & 762.418 & 567.257 & $145.734 ., 7$ & 4.176 .981 \\
alimen $_{i}$ & $\mathrm{R} \$$ & 178 & 318.366 & 339.251 & $23.412,09$ & 3.329 .863 \\
$z_{i}$ & $\mathrm{GgCO}$ eq & 178 & 0,01 & 0,00 & 0,00 & 0,05 \\
\hline
\end{tabular}

Fonte: Resultados da pesquisa.

Para obter as estimativas de eficiência técnica e ambiental, foram estimados dois modelos, um com forma funcional Cobb-Douglas e outro com forma funcional translog, ambos incluindo a variável relativa à emissão de metano como insumo. Procedeu-se, então, o teste de razão de verossimilhança para comparar as duas formas funcionais distintas, e os resultados apontaram o modelo com função de produção translog como a melhor opção ${ }^{3}$. Assim, neste trabalho, serão expostos os resultados obtidos para a função de produção translog, que foi estimada com uma distribuição normal truncada. A Tabela 2 apresenta os valores dos parâmetros estimados para a função translog.

3 O teste indica que o modelo translog está melhor ajustado, em relação ao modelo CobbDouglas, ao nível de significância de 1,8\%. 
Tabela 2. Resultados do modelo de fronteira estocástica para a função de produção translog

\begin{tabular}{|c|c|c|c|}
\hline Variável & Coeficiente & Erro-padrão & $P>|z|$ \\
\hline lnarea $_{i}$ & 0,020 & 0,046 & 0,665 \\
\hline $\ln t r a b_{i}$ & 0,033 & 0,064 & 0,604 \\
\hline $\operatorname{lnalimen}_{i}$ & 0,505 & 0,054 & 0,000 \\
\hline $\ln c a p_{i}$ & 0,176 & 0,080 & 0,029 \\
\hline $\ln z_{i}$ & 0,322 & 0,114 & 0,005 \\
\hline $\ln \left(\text { area }_{i}\right)^{2}$ & $-0,069$ & 0,052 & 0,188 \\
\hline $\operatorname{lnarea}_{i} \times \ln \operatorname{trab}_{i}$ & 0,173 & 0,136 & 0,203 \\
\hline $\operatorname{lnarea}_{i} x$ lnalimen $_{i}$ & $-0,074$ & 0,140 & 0,595 \\
\hline $\operatorname{lnarea}_{i} x \ln$ cap $_{i}$ & $-0,163$ & 0,166 & 0,328 \\
\hline $\ln \operatorname{lrea}_{i} \times \ln z_{i}$ & 0,354 & 0,277 & 0,202 \\
\hline $\ln \left(t r a b_{i}\right)^{2}$ & $-0,413$ & 0,207 & 0,046 \\
\hline $\ln$ trab $_{i} \times$ lnalimen $_{i}$ & 0,303 & 0,256 & 0,236 \\
\hline $\ln t r a b_{i} \times{\ln c a p_{i}}_{i}$ & 0,054 & 0,322 & 0,865 \\
\hline $\ln t r a b_{i} \times \ln z_{i}$ & 0,509 & 0,309 & 0,100 \\
\hline $\ln \left(\text { alimen }_{i}\right)^{2}$ & 0,164 & 0,124 & 0,185 \\
\hline $\operatorname{lnalimen}_{i} \times \operatorname{lncap}_{i}$ & 0,019 & 0,296 & 0,949 \\
\hline $\operatorname{lnalimen}_{i} \times \ln z_{i}$ & $-0,683$ & 0,347 & 0,049 \\
\hline $\ln \left(c a p_{i}\right)^{2}$ & $-0,137$ & 0,099 & 0,166 \\
\hline $\ln \operatorname{cap}_{i} \times \ln z_{i}$ & 0,290 & 0,332 & 0,930 \\
\hline $\ln \left(z_{i}\right)^{2}$ & 0,229 & 0,280 & 0,413 \\
\hline Cons & 0,235 & 0,031 & 0,000 \\
\hline sigma_V & 0,072 & 0,014 & \\
\hline Sigma_u & 0,151 & 0,024 & \\
\hline sigma $^{2}$ & 0,028 & 0,005 & \\
\hline Lambda & 2,076 & 0,036 & \\
\hline
\end{tabular}

Fonte: Resultados da pesquisa.

Realizou-se um teste de hipótese para verificar se o modelo estimado possui ou não componente de ineficiência. A hipótese nula do teste é que $H_{0}: \delta_{u}^{2}=$ 0 , contra a hipótese alternativa de que $H_{1}: \delta_{u}^{2}>0$. Desse modo, caso a hipótese nula não seja rejeitada, o modelo de fronteira estocástica estimado se reduz a um modelo de mínimos quadrados com erros normais. $\mathrm{O}$ teste possui distribuição qui-quadrado e foi realizado no nível de $5 \%$ de significância. $\mathrm{O}$ valor obtido foi de $\mathrm{LR}=4,15$, excedendo o valor crítico de 2,71, e a hipótese nula de que não há ineficiência técnica no sistema de produção leiteiro do Triângulo Mineiro e Alto Paranaíba foi rejeitada.

Ressalta-se que os sinais dos parâmetros estão em conformidade com a literatura acerca do tema e, com exceção das variáveis relativas à despesa com mão de obra e à área da propriedade, as demais variáveis de primeira ordem foram estatisticamente significativas.

Com base na função proposta, foi possível estimar os índices de eficiência técnica de cada unidade da amostra. A Tabela 3 apresenta as estatísticas descritivas do nível de eficiência técnica obtido na estimação. 
Tabela 3. Estatística descritiva da eficiência técnica das fazendas analisadas

\begin{tabular}{cccccc}
\hline Variável & Observação & Média & $\begin{array}{c}\text { Desvio- } \\
\text { padrão }\end{array}$ & Mínimo & Máximo \\
\hline $\begin{array}{c}\text { Eficiência } \\
\text { Técnica }\end{array}$ & 178 & 0,890 & 0,062 & 0,681 & 0,977 \\
\hline
\end{tabular}

Fonte: Resultados da pesquisa.

Os resultados mostram que a eficiência técnica média para as 178 unidades produtivas analisadas foi de 0,89. A propriedade menos eficiente tem eficiência de 0,68. Assim, na Tabela 4, observa-se que cerca de 10,11\% das fazendas operam nos níveis mais baixos de eficiência e 10,67\% das fazendas operam nos níveis de eficiência técnica mais elevados.

Tabela 4. Distribuição das unidades produtivas de acordo com a eficiência técnica

\begin{tabular}{cc}
\hline Intervalo de eficiência técnica & Proporção dentro do intervalo \\
\hline $0,68-0,80$ & $10,11 \%$ \\
$0,80-0,90$ & $34,26 \%$ \\
$0,90-0,95$ & $44,94 \%$ \\
$0,95-1,00$ & $10,67 \%$ \\
\hline
\end{tabular}

Fonte: Resultados da pesquisa.

Reinhard, Lovell e Thijssen (1999) explicam que, quando se encontra resultados que apontam para um nível elevado de eficiência técnica, uma parcela pequena da produção comercializável é perdida devido ao desperdício dos recursos.

Como mencionado anteriormente, a eficiência ambiental foi mensurada com base na eq. (10), ou seja, ela se dá por meio das estimativas dos coeficientes de interação entre a variável ambiental (detrimental input) e os demais insumos (conventional inputs). Assim, os resultados obtidos podem ser observados na Tabela 5. À luz do método utilizado, a média da eficiência ambiental das 178 unidades produtivas avaliadas foi de 0,73.

Tabela 5. Estatística descritiva da eficiência ambiental das fazendas analisadas

\begin{tabular}{cccccc}
\hline Variável & Observações & Média & $\begin{array}{c}\text { Desvio- } \\
\text { padrão }\end{array}$ & Mínimo & Máximo \\
\hline $\begin{array}{c}\text { Eficiência } \\
\text { Ambiental }\end{array}$ & 178 & 0,733 & 0,148 & 0,273 & 0,957 \\
\hline
\end{tabular}

Fonte: Resultados da pesquisa.

Ressalta-se que a eficiência ambiental apresentou alta dispersão entre as unidades produtivas avaliadas. Ao observar a Tabela 6, verifica-se que cerca 
de 7,86\% das propriedades apresentaram índice de eficiência ambiental maior que $90 \%$, enquanto $8,98 \%$ apresentaram valores inferiores a $50 \%$.

\section{Tabela 6. Distribuição das unidades produtivas de acordo com a eficiência ambiental}

\begin{tabular}{cc}
\hline Eficiência Ambiental & Distribuição Percentual \\
\hline $0,00-0,50$ & $8,98 \%$ \\
$0,50-0,70$ & $24,15 \%$ \\
$0,70-0,80$ & $29,21 \%$ \\
$0,80-0,90$ & $29,77 \%$ \\
$0,90-1,00$ & $7,86 \%$ \\
\hline
\end{tabular}

Fonte: Resultados da pesquisa.

Primavesi et al. (2004) destacam que, para reduzir a emissão de metano por quilograma de leite e, por conseguinte, melhorar o desempenho da eficiência ambiental, é necessário aprimorar a dieta do rebanho. Para tal, é preciso fornecer ao animal alimentos com maiores índices de energias digeríveis, com maiores conteúdos de proteína e menos fibrosos. Além disso, é imprescindível investir na melhoria das pastagens e na suplementação alimentar fornecida ao rebanho e melhorar o manejo da produção (PRIMAVESI et al., 2004).

Foi realizado também um teste de correlação entre a eficiência ambiental e a eficiência técnica das fazendas analisadas. Assim, verificou-se, através da correlação de Spearman, que há uma correlação positiva entre as duas eficiências, de 0,812, que é significativa a 5\%. De acordo com Dayananda (2016), a correlação positiva entre as duas eficiências pode ser em parte explicada pelos meios adotados para se reduzir as emissões de GEE. Conforme já mencionado, parcela expressiva das emissões de metano liberado pelo rebanho bovino leiteiro no Brasil ocorre devido ao processo de fermentação entérica. Dessa forma, a redução no uso de ração para um determinado rendimento de leite aumenta a eficiência técnica da fazenda, do mesmo modo que a diminuição de insumos emissores de metano melhora a eficiência ambiental da propriedade. Assim, dado que a fermentação entérica é a principal fonte de emissão de gases de efeito estufa, ao melhorar a alimentação do rebanho, desenvolve-se tanto a eficiência técnica quanto a ambiental, o que resulta na correlação positiva entre as duas variáveis.

Desse modo, essas medidas, além de proporcionarem redução da emissão de metano, também gerariam melhorias no desempenho produtivo. A correlação positiva entre a eficiência ambiental e a técnica encontrada neste estudo indica que ambas podem ser melhoradas em conjunto. Os resultados obtidos por Reinhard, Lovell e Thijssen (1999), Tamini et al. (2011) e Dayananda (2016), que, através do índice de correlação de Spearman, estimaram a correlação entre a eficiência técnica e a ambiental, apontaram índices de 0,87, 0,71 e 0,41, respectivamente. 
A eficiência ambiental encontrada neste estudo foi de $89,0 \%$ em média. A estimativa de eficiência técnica, neste trabalho, foi semelhante ao obtido por Reinhard, Lovell e Thijssen (1999), que também obtiveram média de 89\% para as fazendas leiteiras da Holanda. O valor obtido por Dayananda (2016), por sua vez, foi de $82 \%$. Já a estimativa de eficiência técnica encontrada por Tamini et al. (2011), foi inferior à dos demais autores, sendo de $42 \%$.

O resultado da eficiência técnica obtido nesta pesquisa também foi superior ao dos demais estudos que analisaram somente o índice de eficiência técnica para o setor leiteiro em Minas Gerais. Nascimento et al. (2012), ao estimarem a eficiência técnica das fazendas leiteiras de Minas, encontraram um valor médio de $82,6 \%$. Os autores observaram uma grande assimetria na distribuição do índice de eficiência entre as propriedades. A produção de leite no estado, como já mencionado, é caracterizada por muitas diferenças entre as mesorregiões. Dessa forma, pode ser que a heterogeneidade das regiões mineiras explique a assimetria encontrada pelos autores.

No caso específico deste estudo, cabe ressaltar que o alto índice de eficiência média encontrado pode ser explicado pelo fato de as fazendas analisadas serem participantes do projeto Educampo. Dessa forma, acredita-se que essas propriedades possuem um alto nível tecnológico, pois elas recebem assistência técnica periódica, incorporando tecnologias e práticas de manejo que resultam em maior produtividade técnica e econômica.

Outro fator que pode explicar o nível de produtividade observado neste trabalho é a localização das fazendas analisadas. Nos últimos anos, como já citado, a mesorregião do TM/AP se tornou a principal bacia leiteira do estado, apresentando maior crescimento e maior produtividade em relação às outras regiões. Ao comparar a produtividade nas mesorregiões mineiras, observa-se que a região composta pelo Triângulo Mineiro e Alto Paranaíba apresenta a maior do estado, com 1,92 litros/vaca/ano. Em seguida, destaca-se o Sul e Sudoeste mineiro, com produtividade igual a 1,77 litros/vaca/ano.

Em relação à eficiência ambiental encontrada neste estudo, estimada em média em $73,4 \%$, esta foi superior às estimativas internacionais realizadas por Reinhard, Lovell e Thijssen (1999) e Dayananda (2016), que obtiveram valores de $44 \%$ e $30 \%$, respectivamente. ${ }^{4}$

Campos (2011) também encontrou médias inferiores às deste estudo para a eficiência ambiental. As propriedades que trabalham com sistemas extensivos apresentaram uma eficiência ambiental baixa, de cerca de 13,7\%, com retornos constantes à escala, e 15,9\%, com retornos variáveis. Em contrapartida, as fazendas com sistema intensivo obtiveram média igual a $11,1 \%$, com retornos constantes à escala, e 23\%, com retornos variáveis.

\footnotetext{
${ }^{4}$ Cabe reiterar que, embora os autores tenham usado o mesmo método proposto neste estudo, utilizaram o problema ambiental da nitrificação do solo, e não a fermentação entérica, para medir a eficiência ambiental, e aplicaram seus modelos para outros países, o que torna os resultados não totalmente comparáveis.
} 
Por fim, Dayananda (2016) afirma que, quanto maior a produção média de leite do rebanho, menor será a intensidade das emissões de GEE. Assim, vacas altamente produtivas geram uma quantidade menor de metano por unidade de leite produzido.

\section{CONSIDERAÇÕES FINAIS}

A média da eficiência técnica das fazendas analisadas neste trabalho foi de 0,89 . Todas as variáveis tratadas no modelo obtiveram os sinais esperados e, com exceção da despesa com mão de obra e da área da propriedade, todas os insumos considerados foram estatisticamente significativos. A variável relativa à despesa com alimento é a que provoca o maior impacto na quantidade produzida.

A estimativa da eficiência ambiental encontrada neste trabalho foi de 0,733 . Essa eficiência apresentou uma variabilidade maior que a da eficiência técnica entre as fazendas analisadas, mostrando que as eficiências ambientais das unidades produtoras são heterogêneas.

A correlação entre a eficiência técnica e a ambiental, medida pela correlação de Spearman, foi positiva. Esse resultado indicou que propriedades que apresentaram uma maior eficiência técnica também apresentaram uma maior eficiência ambiental.

No decorrer desta pesquisa, foram encontradas diversas limitações. Entre elas, a indisponibilidade de dados detalhados referentes à alimentação e à idade do rebanho. Para melhor avaliar a emissão de gases de efeito estufa por ruminantes, seria necessário ter informações sobre a quantidade de alimento ingerido pelos animais e a faixa etária deles. De posse dessas informações, também seria possível calcular os efeitos marginais sobre o índice de eficiência ambiental. Saber o que o animal ingere e a qualidade de sua alimentação afeta a emissão de metano através da fermentação entérica. Apesar das limitações encontradas, foi possível calcular a quantidade de emissão de metano utilizando dados disponibilizados pelo IPCC (2006) e MCTI (2010). Visando à melhoria dos índices de eficiência técnica e ambiental, é necessário melhorar a alimentação do rebanho, a área de pastagem e a tecnologia utilizada na produção.

Para futuros trabalhos, recomenda-se analisar o tipo e a quantidade de alimentação do rebanho. Além disso, sugere-se estudar a emissão de metano via manejo de dejetos, visto que a decomposição anaeróbica dos dejetos eliminados por bovinos produz quantidades expressivas de metano. Ademais, sugere-se que sejam realizados estudos relacionados a possíveis políticas públicas que incentivem os produtores de leite a produzir de maneira mais eficiente, tanto em relação a aspectos técnicos quanto ambientais. Do ponto de vista da metodologia, trabalhos futuros poderiam usar métodos de fronteira estocástica, tratando a emissão de gases como produto indesejável ao invés de tratá-la como insumo. Esse procedimento traria maior grau de realismo à modelagem empregada. 


\section{AGRADECIMENTOS}

O presente trabalho foi realizado com apoio da Coordenação de Aperfeiçoamento de Pessoal de Nível Superior - Brasil (CAPES) - Código de Financiamento 001.

\section{REFERÊNCIAS}

AIGNER, D.; LOVELL, C.A.K.; SCHMIDT, P. Formulation and estimation of stochastic frontier production function models. Journal of Econometrics. $\mathrm{v}$. 6, n. 1, p. 21-37, 1977. <doi: 10.1016/0304-4076(77)90052-5>.

BARUA, M.O.; BROCKETT, P.L.; COOPER, W.W.; DENG, H.; PANKER, B.R.; RUEFLI, T.W.; WHINSTON, A. DEA evaluations of long - and shortrun efficiencies of digital vs. physical product "dot com" companies. SocioEconomic Planning Sciences. v. 38, n. 4, p. 233-253, 2004. doi: 10.1016/j.seps.2003.10.002.

BATTESE, G.E.; COELLI, T.J. Frontier production functions, technical efficiency and panel data: with application to Paddy farmers in India. Journal of Productivity Analysis, v. 3, p. 153-169. 1992. < doi:10.1016/03044076(77)90052-5>.

BERRE, D.; BLANCARD, S.; BOUSSEMART, J-P.; LELEU, H.; TILLARD, E. Finding the right compromise between productivity and environmental efficiency on high input tropical dairy farms: a case study. Journal of Environmental Management, 235-244, $2014 . \quad$ doi: 10.1016/j.jenvman.2014.07.008.

CAMPOS, S. A. C. Eficiência econômica e ambiental da produção leiteira em Minas Gerais. 2011. 171 f. Dissertação (Mestrado em Economia Aplicada) Universidade Federal de Viçosa, Viçosa. Disponível em: <http://locus.ufv.br/handle/123456789/51>. Acesso em: 03 dez. 2016.

CARDOSO, A.S. Avaliação das emissões de gases de efeito estufa em diferentes cenários de intensificação de uso das pastagens no Brasil Central. 2012. $99 \mathrm{f}$. Dissertação (Mestrado em Ciências) - Instituto de Agronomia, Universidade Federal Rural do Rio de Janeiro, Seropédica. Disponível em: <http://www.cnpc.org.br/arquivos/avaemgaefesdiceinuspabracen.pdf >. Acesso em: 13 ago. 2016.

CARVALHO, L.A. et al. Importância econômica do leite. Sistema de Produção 2. Embrapa gado de Leite, 2003. Disponível em: <https://sistemasdeproducao.cnptia.embrapa.br/FontesHTML/Leite/Lei teCerrado/importancia.html>. Acesso em: 23 fev. 2016.

CENTRO DE ESTUDOS AVANÇADOS EM ECONOMIA APLICADA CEPEA. PIB Agro Minas Gerais. CEPEA-USP. Disponível em: <http:/ / www.cepea.esalxq.usp.br/br/pib-do-agronegocio-de-minasgerais.aspx>. Acesso em: 22 out. 2016. 
CENTRO DE ESTUDOS AVANÇADOS EM ECONOMIA APLICADA CEPEA. PIB Agro. CEPEA-USP, 1995/2015. Disponível em: <http://www.cepea.esalq.usp.br/br/pib-do-agronegociobrasileiro.aspx>. Acesso em: 18 out. 2016.

COELLI, T.J.. Recent developments in frontier modelling and efficiency measurement. Australian Journal of Agricultural Economics, v. 39, n. 3, p. 219245, 1995. doi: <10.1111/j.1467-8489.1995.tb00552.x>.

COELLI, T.; LAUWERS, L.; HUYLENBROECK, G.V. Environmental efficiency meansurement and the materials balance condition. Journal of Productivity Analysis, v. 28, n.1-2, p. 2-12, 2006. doi:<10.1007/s11123-0070052-8>.

DAYANANDA, C. Technical and environmental efficiencies of Ontario dairy farming. 2016. 108 f. Dissertação (Mestrado em Ciências) - University of Guelph, Ontario. Disponível em: <https://atrium.lib.uoguelph.ca/xmlui/bitstream/handle/10214/10026/ Dayananda_Chathurika_201609_Msc.pdf?sequence=1\&isAllowed=y $>$. Acesso em: 18 mar. 2017.

FÄRE, R.; GROSSKOPF, S.; NOH, D. W.; Weber, W. Characteristics of a polluting technology: theory and practice. Journal of Econometrics, v. 126, n. 2, p. 469-492, 2005. doi: <10.1016/j.jeconom.2004.05.010>.

FUNDAÇÃO ESTADUAL DO MEIO AMBIENTE - FEAM. Estimativas de emissões e remoções de gases de efeito estufa do estado de Minas Gerais - 2014. Belo Horizonte, 2016. Disponível em: <http://pemc.meioambiente.mg.gov.br/images/Estimativas_GEE_2005_ 2014_MG_FEAM_v02-1.pdf>. Acesso em: 25 jan. 2017.

FUNDAÇÃO INSTITUTO BRASILEIRO DE GEOGRAFIA E ESTATÍSTICA - IBGE. Censo Agropecuário 2006. Rio de Janeiro, 2009. Disponível em: $<$ http://biblioteca.ibge.gov.br/visualizacao/periodicos/51/agro_2006.pd f>. Acesso em: 13 ago. 2015.

FUNDAÇÃO INSTITUTO BRASILEIRO DE GEOGRAFIA E ESTATÍSTICA - IBGE. Pesquisa Pecuária Municipal 2016. SIDRA (Sistema Brasileiro de Recuperação Automática). Disponível em: <http://www.sidra.ibge.gov.br/>. Acesso em: 10 nov. 2017.

GOURLEY, C.J.P; AARONS, S.R.; POWEL, J.M. Nitrogen use efficiency and manure management practices in contrasting dairy production systems. Agriculture, Ecosystems and Environment, v. 147, p. 73-81. 2012. doi:10.1016/j.agee.2011.05.011.

HAILU, A.; VEEMAN, T.S. Non-parametric productivity analysis with undesirable outputs: an application to the Canadian pulp and paper industry. American Journal of Agricultural Economics, v. 83, n. 3, p. 605-616, 2001. doi: 10.1111/0002-9092.00181.

INTERNATIONAL FARM COMPARISON NETWORK - IFCN. Word Dairy Map 2014. Kiel: IFCN, 2014. Disponível em: <http://www.ifcndairy.org/en/start/index.php>. Acesso em: 15 jan. 2017. 
INTERGOVERNMENTAL PANEL ON CLIMATE CHANGE - IPCC. Guidelines for National Greenhouse Gas Inventories. Agriculture, Forestry and Other Land Use - Emissions from Livestock and Manure Management. v. 4, cap 10. 2006. Disponível em: <http://www.ipccnggip.iges.or.jp/public/2006gl/pdf/4_Volume4/V4_10_Ch10_Livestock. pdf>. Acesso em: 23 mar. 2017.

KUMBHAKAR, S. C.; LOVELL, C. A. K. Stochastic frontier analysis. Cambridge: Cambridge University Press, 2000. doi: $\underline{10.1017 / C B O 9781139174411 .}$

LANSINK, A.O.; PIETOLA, K.; BÄCKMAN, S. Effciency and productivity of conventional and organic farms in Finland 1994-1997. European Review of Agricultural Economics, v. 29, n. 1, p. 51-65, 2002. doi: 10.1093/erae/29.1.51.

LEE, J.D.; PARK, J.B.; KIM, T.Y. Estimation of the shadow prices of pollutants with production/environment inefficiency taken into account: a nonparametric directional distance function approach. Journal of environmental management, v. 64, n. 4, p. 365-375, 2002 doi: 10.1006/jema.2001.0480.

LIMA, A.L.R. Eficiência produtiva e econômica da atividade leiteira em Minas Gerais. 2006. 77 f. Dissertação (Mestrado em Administração) - Universidade Federal de Lavras, Lavras. Disponível em: <http://repositorio.ufla.br/bitstream/1/1995/1/DISSERTA\%C3\%87\%C3 \%830_Efici\%C3\%AAncia\%20produtiva\%20e\%20econ\%C3\%B4mica \%20da \%20atividade\%20leiteira \%20em\%20Minas\%20Gerais.pdf>. Acesso em: 29 dez. 2016.

MINISTÉRIO DA CIÊNCIA, TECNOLOGIA e INOVAÇÃO - MCTI. Emissões de metano por fermentação entérica e manejo de dejetos de animais. Segundo inventário brasileiro de emissões antrópicas de gases de efeito estufa. Brasília, 2010. Disponível em: <http://www.mct.gov.br/upd_blob/0235/235580.pdf>. Acesso em: 24 ago. 2016.

MINISTÉRIO DA CIÊNCIA, TECNOLOGIA e INOVAÇÃO - MCTI. Estimativas anuais de emissões de gases de efeito estufa. 2 ed. Brasília, 2014. Disponível em: <http://sirene.mcti.gov.br/documents/1686653/1706227/LIVRO_MCTI C_EstimativaDeGases_Publica\%C3\%A7\%C3\%A30_210x297mm_FINAL_ WEB.pdf/61e78a4d-5ebe-49cd-bd16-4ebca30ad6cd>. Acesso em: 10 fev. 2016.

MINISTÉRIO DA CIÊNCIA, TECNOLOGIA e INOVAÇÃO - MCTI. Estimativas anuais de emissões de gases de efeito estufa. 3 ed. Brasília, 2016. Disponível em: <http://sirene.mcti.gov.br/documents/1686653/1706227/LIVRO_MCTI C_EstimativaDeGases_Publica\%C3\%A7\%C3\%A30_210x297mm_FINAL_ WEB.pdf/61e78a4d-5ebe-49cd-bd16-4ebca30ad6cd>. Acesso em: 05 jan. 2017. 
NASCIMENTO A.C.C., LIMA J.E.D., BRAGA M.J., NASCIMENTO M., GOMES A.P. Eficiência técnica da atividade leiteira em Minas Gerais: uma aplicação de regressão quantílica. Revista Brasileira de Zootecnia, v. 41, n.3, p. 783-789, 2012. doi: 10.1590/S1516-35982012000300043.

NIJUKI, E.; BRAVO-URETA, B. E. The economic costs of environmental regulation in U.S. dairy farming: a directional distance function approach. American Journal of Agricultural Economics, v. 97, n. 4, p. 1087-1106, 2015. < doi: 10.1093/ajae/aav007>.

PRIMAVESI, O.; FRIGHETTO, R.T.S.; PEDREIRA, M.S.; LIMA, M.A.; BERCHIELLI, T.T.; BARBOSA, P.F. Metano entérico de bovinos leiteiros em condições tropicais brasileiras. Pesquisa agropecuária brasileira, v.39, n.3, p.277-283, 2004. doi: 10.1590/S0100-204X2004000300011.

PRIMAVESI, O; PEDREIRA, M; FRIGHETTOE, R; LIMA, M.A; BERCHIELLI, T.T, OLIVEIRA, S.G, RODRIGUES, A.D.A, BARBOSA, P.F. Manejo alimentar de bovinos leiteiros e sua relação com produção de metano ruminal. São Carlos: Embrapa Pecuária Sudeste, 2004. 21 p. (Embrapa Pecuária Sudeste. Circular Técnica, 39). Disponível em: <https://www.infoteca.cnptia.embrapa.br/bitstream/doc/47010/1/Circ ular39.pdf>. Acesso em: 15 out. 2016.

REINHARD, S.; LOVELL, C.A.K.; THIJSSEN, G. Econometric estimation of technical and environmental efficiency: an application to dutch dairy farms. American Journal of Agricultural Economics, v. 81, n. 1, p. 44-60, 1999. < doi: $\underline{10.2307 / 1244449>}$.

REINHARD, S.; LOVELL, C.A.K.; THIJSSEN, G.J. Environmental efficiency with multiple environmentally detrimental variables; estimated with SFA and DEA. European Journal of Operational Research, v. 121, n. 2, p. 287-303, 2000. doi: 10.1016/S0377-2217(99)00218-0.

TAMINI, L.D.; LARUE, B. WEST, G. Technical and environmental efficiencies and best management practices in agriculture. Applied Economics, v. 44, n. 13, p. 1659-1672, 2011. doi: 10.1080/00036846.2010.548789. 